\title{
CMEs and Long-Lived Geomagnetic Storms: A Case Study
}

\author{
H. Xie ${ }^{1,2}$, N. Gopalswamy ${ }^{2}$, P.K. Manoharan ${ }^{3}$, \\ S. Yashiro ${ }^{1,2}$, A. Lara ${ }^{4}$, and S. Lepri ${ }^{5}$ \\ ${ }^{1}$ The Catholic University of America, Washington DC, USA \\ ${ }^{2}$ NASA Goddard Space Flight Center, Greenbelt, Maryland, USA \\ ${ }^{3}$ National Center for Radio Astronomy, Ooty, INDIA \\ ${ }^{4}$ Instituto de Geofisica, UNAM, Mexico \\ ${ }^{5}$ University of Michigan, Michigan, USA
}

\begin{abstract}
We studied the relationship between successive coronal mass ejections (CMEs) and a long-lived geomagnetic storm (LLGMS) by examining the 1998 May 4 event. Five successive CMEs from the same active region and four interplanetary shocks were found to be associated with this LLGMS. We investigated the effect of successive and interacting CMEs on the LLGMS.
\end{abstract}

Keywords. Sun: coronal mass ejections (CMEs), solar-terrestrial relations.

\section{Introduction}

It is now well established that front-side halo coronal mass ejections (CMEs) are the major cause for large geomagnetic storms (e.g., Burlaga et al. 2002; Cane et al. 2000; Gopalswamy et al. 2000; Webb et al. 2000; Zhang et al. 2003;). Isolated geomagnetic storms typically have a recovery phase less than $\sim 1$ day. Some storms have main and recovery phases exceeding $\sim 3$ days. We call them long-lived geomagnetic storms (LLGMS). LLGMSs occur mostly when successive CMEs ejected from the Sun and impact Earth. In this paper, we present a case study of a LLGMS involving successive CMEs and CME interaction.

\section{Data and models}

The LLGMS of interest as defined by the Dst (disturbance storm time) index, and reported by the World Data Center in Kyoto (http://swdcwww.kugi.kyoto-u.ac.jp/dstdir/) was observed from 1998 May 2 - 7. We use Fe charge state data and the component of the interplanetary magnetic field $B_{z}$ to help identify the interplanetary CMEs (ICMEs). The associated CMEs were from the catalog of CMEs observed by the Solar and Heliospheric Observatory (SOHO) mission's coronagraphs (http://cdaw.gsfc.nasa.gov/CME_list). The height-time profiles of CMEs were plotted to examine if there was any possible interaction between successive CMEs.

Figure 1(a) shows the Dst, Fe charge state $\left(Q_{F e}\right), B_{z}$, and the height-time profiles of the associated CMEs for the 1998 May 4 event. The LLGMS lasted for 5 days from May 2 to May 7 (main phase $\sim 2$ days and recovery phase $\sim 3$ days). The $D_{s t_{\text {min }}}$ was $\sim-205$ $\mathrm{nT}$.

Five CMEs were found to be associated with the LLGMS. The CMEs originated from AR8210 when it was at S17E23, S18W05, S20W07, S15W15, and S13W34. From CME height-time profiles, we can see that CME 1 and CME 5 were well separated, but CME 2, CME 3, and CME 4 were ejected in quick succession. The speeds of CME 2 and CME 3 
(a)
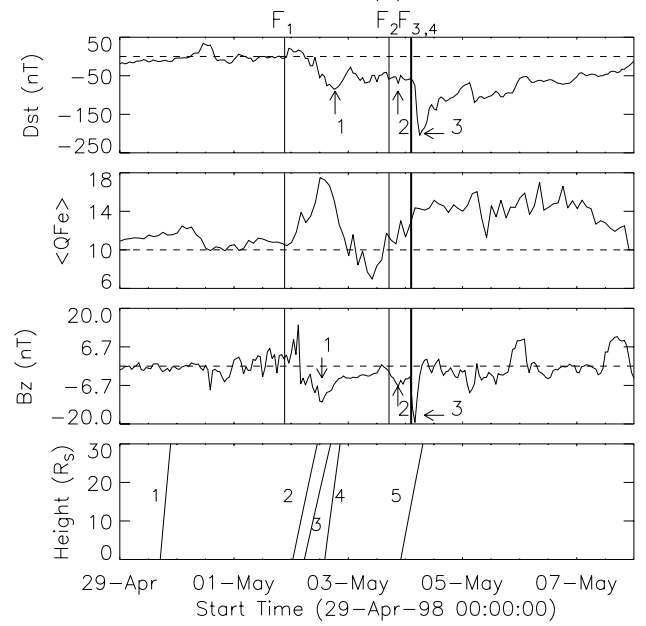

(b)
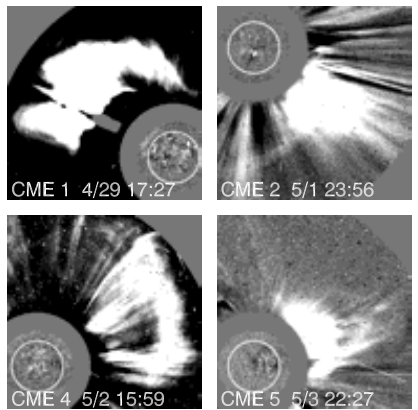

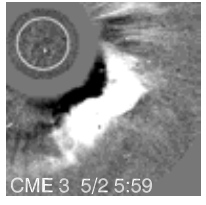

CME Speed $(\mathrm{km} / \mathrm{s})$

1(Halo) 1374

2(Halo) 585

3(Halo) 542

4(Halo) 938

5(Partial) 649

Figure 1. (a) The Dst, $Q_{F e}, B_{z}$, and CME height-time profiles. The vertical solid lines $\left(F_{1}, F_{2}\right.$, $\left.F_{3}, F_{4}\right)$ indicate the ICME shock fronts. Note that $F_{3}$ and $F_{4}$ are very close to each other, and the drop in $Q_{F e} \sim 6$ before $F_{2}$ is due to the instrumental noise. The numbers on the height-time plots indicate the associated CMEs. (b) The five CMEs associated with the LLGMS: C2 images superposed with EIT images. The times and speeds of the CMEs are indicated.

were 585 and $542(\mathrm{~km} / \mathrm{s})$, respectively, and their trajectories were nearly parallel. CME 4 was faster $(938 \mathrm{~km} / \mathrm{s})$ than CME 2 and CME 3 . It is likely that CME 4 would catch up with CME 3, causing CME 3 to speed up and CME 4 to slow down, thus producing two successive strong shocks $F_{3}$ and $F_{4}$. Possible interaction occurred between CME 3 and CME 4, but they were not totally merged at 1 AU. CME 3 and CME 4 were separated by $\sim 8.5$ hrs near the Sun. But they were separated by only $\sim 0.5$ hrs at 1 AU. Four shocks shown in the figure are related to CME 1, CME 2, and possible interaction of CME 3 and CME 4, respectively.

Three dips in the main phase of the storm and in the $B_{z}$ profile can be seen in Fig. 1 (pointed by arrows): the first related to the interplanetary MC associated with CME 1, the second related to CME 2, and the last $D S T_{\text {min }}$ caused by the possible interaction of CME 3 and CME 4. Successive CMEs increase the duration of LLGMS. CME interaction enhances the intensity of LLGMS. However, our statistical study indicates that successive interacting CMEs can also lead to modest LLGMS (details will be presented elsewhere).

\section{Acknowledgements}

This research was supported by NASA LWS and NSF SHINE (ATM 0204558) programs.

\section{References}

Burlaga, L.F., Plunkett, S.P., St., \& Cyr, O.C. 2002, J. Geophys. Res. 107, SSH 1-1

Cane, H.V., Richardson, I.G., St., \& Cyr, O.C. 2000, Geophys. Res. Lett. 27, 3591

Gopalswamy, N., Lara, A., Lepping, R.P., Kaiser, M.L., Berdichevsky, D., \& St. Cyr, O.C. 2000, Geophys. Res. Lett. 27, 145

Webb, D.F., Cliver, E.W., Crooker, N.U., St., Cry, O.C., \& Thompson, B. 2002, J. Geophys. Res. 105, 7491

Zhang, J., Dere, K.P., Howard, R.A., \& Bothmer, V. 2003, Astrophys. J. 582, 520 